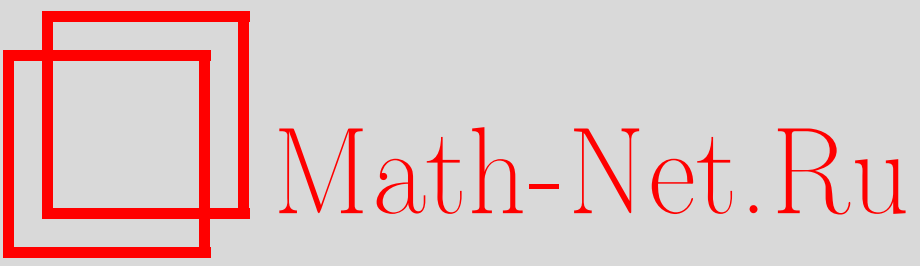

Ю. П. Вирченко, А. В. Субботин, Гиперболические квазилинейные ковариантные уравнения первого порядка

дивергентного типа для векторного поля на $\mathbb{R}^{3}$, Итоги науки и техн. Сер. Соврем. мат. и ее прил. Темат. обз., 2021, том 191, 16-28

DOI: https://doi.org/10.36535/0233-6723-2021-191-16-28

Использование Общероссийского математического портала Math-Net.Ru подразумевает, что вы прочитали и согласны с пользовательским соглашением

http://www. mathnet.ru/rus/agreement

Параметры загрузки:

IP : 18.208 .226 .222

26 апреля 2023 г., 16:04:17 
ИТОГИ НАУКИ И ТЕХНИКИ.

Современная математика и ее приложения.

Тематические обзоры.

Том 191 (2021). С. $16-28$

DOI: 10.36535/0233-6723-2021-191-16-28

УДК 517.952.1; 517.956.3

\title{
ГИПЕРБОЛИЧЕСКИЕ КВАЗИЛИНЕЙНЫЕ КОВАРИАНТНЫЕ УРАВНЕНИЯ ПЕРВОГО ПОРЯДКА ДИВЕРГЕНТНОГО ТИПА ДЛЯ ВЕКТОРНОГО ПОЛЯ НА $\mathbb{R}^{3}$
}

\author{
() 2021 г. Ю. П. ВИРЧЕНКО, А. В. СУББОТИН
}

\begin{abstract}
АннотАция. В работе представлено полное описание класса гиперболических квазилинейных уравнений первого порядка дивергентного типа, описывающих изменение при $t \in \mathbb{R}$ векторных полей $\boldsymbol{v}(\boldsymbol{x}, t), \boldsymbol{x} \in \mathbb{R}^{3}$, которые инвариантны относительно трансляций времени $t \in \mathbb{R}$ и пространства $\mathbb{R}^{3}$, а также преобразующихся ковариантным образом при преобразованиях группы $\mathbb{O}_{3}$ вращений $\mathbb{R}^{3}$. Проведено сравнение этого класса с классом аналогичных уравнений, гиперболических по Фридрихсу.
\end{abstract}

Ключевъе слова: квазилинейная система уравнений, уравнение дивергентного типа, гиперболичность, трансляционная инвариантность, векторное поле, ковариантность, плотность потока поля.

\section{HYPERBOLIC QUASILINEAR COVARIANT FIRST-ORDER EQUATIONS OF DIVERGENT TYPE FOR VECTOR FIELDS ON $\mathbb{R}^{3}$}

\author{
(c) 2021 YU. P. VIRCHENKO, A. V. SUBBOTIN
}

\begin{abstract}
In this paper, we present a complete description of the class of first-order hyperbolic quasilinear equations of divergent type that describe the change in time $t \in \mathbb{R}$ of vector fields $\boldsymbol{v}(\boldsymbol{x}, t)$, $\boldsymbol{x} \in \mathbb{R}^{3}$, which are invariant under translations in time $t \in \mathbb{R}$ and space $\mathbb{R}^{3}$ and transform covariantly under transformations from the group $\mathbb{O}_{3}$ of rotations of the space $\mathbb{R}^{3}$. This class is compared with the class of similar equations, which are hyperbolic in the sense of Friedrichs.
\end{abstract}

Keywords and phrases: quasilinear system, equation of divergent type, hyperbolicity, translational invariance, vector field, covariance, flux density.

AMS Subject Classification: 35L40, 35L60

1. Введение. В $[2,3,11]$, с целью решения принципиальных задач неравновесной термодинамики конденсированных сред была поставлена задача об описании классов систем дифференциальных уравнений дивергентного типа, описывающих эволюцию фиксированного множества полей на евклидовом пространстве на основе таких уравнений, которые удовлетворяют определенным физически естественным условиям. А именно, такого рода уравнения, в отсутствие внешних воздействий, обязаны быть инвариантными относительно группы трансляций времени и группы трансляций пространства $\mathbb{R}^{3}$. Кроме того, они должны преобразовываться специальным образом при вращениях $\mathbb{R}^{3}$. В простейшем случае, который мы далее будем называть сферически симметричным, и который является объектом изучения в настоящей работе, они должны преобразовываться ковариантным образом при действии элементов группы $\mathbb{O}_{3}$, как ее тензорные представления (см., например, [8]). Однако в этих работах не ставился вопрос об обладании решениями конструируемых систем дифференциальных уравнений физически «разумных» свойств, 
необходимых для возможности их использования при моделировании эволюции тех физических систем, для которых они, собственно, предназначены. Настоящая работа посвящена нахождению таких условий для элементов класса $\mathfrak{K}_{1}\left(\mathbb{R}^{3}\right)$, состоящего из квазилинейных уравнений первого порядка дивергентного типа, которые описывают векторное поле $\boldsymbol{v}(\boldsymbol{x}, t), \boldsymbol{x} \in \mathbb{R}^{3}$, при изменении $t \in \mathbb{R}$, и таких, которые удовлетворяют указанным выше условиям, а именно, коэффициенты этих уравнений не зависят явно ни от $t$, ни от $\boldsymbol{x}$, а сами уравнения преобразуются при вращениях $\mathbb{R}^{3}$ как векторы в $\mathbb{R}^{3}$.

Эволюционные уравнения первого порядка, которые используются при описании сплошных сред, как правило, не описывают влияния на их динамику свойственных им физических диссипативных механизмов. В связи с этим физически разумными представляются системы уравнений первого порядка уравнений гиперболического типа (см., например, $[5,10])$. С физической тоски зрения требование гиперболичности системы уравнений первого порядка означает, что все решения $\omega_{j}(\boldsymbol{k}), j=1,2,3$, соответствующего ей «дисперсионного» уравнения для зависимости частоты изменения во времени поля $\boldsymbol{v}(\boldsymbol{x}, t)$ от волнового вектора $\boldsymbol{k} \in \mathbb{R}^{3}$ являются вещественными. В настоящей работе мы установим необходимые и достаточные условия гиперболичности для систем уравнений первого порядка указанного выше типа.

2. Ковариантные векторные уравнения. Рассмотрим эволюционное уравнение дивергентного типа для векторного поля $\boldsymbol{v}(\boldsymbol{x}, t), \boldsymbol{x} \in \mathbb{R}^{3}, t \in \mathbb{R}$, которое имеет вид

$$
\dot{v}_{j}(\boldsymbol{x}, t)=\left(\nabla_{k} S_{j k}[\boldsymbol{v}]\right)(\boldsymbol{x}, t), \quad j=1,2,3
$$

$\left(\nabla_{j} \equiv \partial / \partial x_{j}, j=1,2,3\right)$, где $S_{j k}[\boldsymbol{v}]$ - функция от значений этого поля в текущей пространственно временной точке $\langle\boldsymbol{x}, t\rangle$. В формуле (1) и далее используется правило тензорной алгебры, согласно которому наличие повторяющихся векторных индексов (в данном случае, нижних индексов $k$ ) подразумевает суммирование по всем их допустимым значениям 1, 2, 3. Таким образом, уравнение (1) является уравнением первого порядка и представляет собой систему трех квазилинейных уравнений.

Сформулируем требование ковариантности уравнения (1) относительно его преобразований посредством ортогональных матриц $U$, составляющих группу $\mathbb{O}_{3}$. При поворотах пространства $\mathbb{R}^{3}$, описываемых посредством этих матриц, каждый радиус-вектор $\boldsymbol{x}$ переходит в вектор $\boldsymbol{x}^{\prime}=\boldsymbol{U}_{\boldsymbol{x}}$ При этом значение матриц-функции $\left(S_{j k}[\boldsymbol{v}]\right)(\boldsymbol{x}, t), j, k=1,2,3$, переходит в $\left(S_{j k}\left[\boldsymbol{v}^{\prime}\right]\right)\left(\boldsymbol{x}^{\prime}, t\right)=$ $\left(S_{j k}[\mathrm{U} \boldsymbol{v}]\right)(\mathrm{U} \boldsymbol{x}, t)$ с реализациями векторного поля $\boldsymbol{v}^{\prime}\left(\boldsymbol{x}^{\prime}, t\right)=\boldsymbol{U} \boldsymbol{v}(\mathrm{U} \boldsymbol{x}, t)$. Тогда после поворота пространства уравнение (1) преобразуется в следующее

$$
\dot{v}_{j}^{\prime}(\boldsymbol{x}, t)=\left(\nabla_{k}^{\prime} S_{j k}\left[\boldsymbol{v}^{\prime}\right]\right)\left(\boldsymbol{x}^{\prime}, t\right), \quad j=1,2,3,
$$

где $\nabla_{k}^{\prime}(\cdot)=\left(\nabla_{k}^{\prime} x_{l}\right) \cdot \nabla_{l}(\cdot)=U_{k l} \nabla_{l}(\cdot)$, так как для ортогональных матриц $\left(\mathrm{U}^{-1}\right)_{l k}=U_{k l} \equiv(\mathrm{U})_{k l}$. После подстановки явных выражений для $\nabla_{k}^{\prime} x_{l}=U_{k l}$ и функции $S_{j k}\left[\boldsymbol{v}^{\prime}\right]$ уравнение $(2)$ превращается в уравнение

$$
\dot{v}_{m}(\boldsymbol{x}, t)=\nabla_{l}\left(U_{j m} U_{k l} S_{j k}[\mathrm{U} \boldsymbol{v}]\right)(\boldsymbol{x}, t), \quad j=1,2,3 .
$$

Требование ковариантности состоит в том, что уравнение (2) должно быть идентично уравнению (1), т.е. множества их решений должны совпадать. Это требование приводит к следующему условию на выбор функции $S_{j k}[\boldsymbol{v}]$ :

$$
U_{j m} U_{k l} S_{m l}[\boldsymbol{v}]=S_{j k}[\mathrm{U} \boldsymbol{v}] .
$$

Обозначим посредством $\mathfrak{K}_{1}\left(\mathbb{R}^{3}\right)$ класс уравнений $(1)$ с матриц-функциями $S_{j k}[\boldsymbol{v}]$, которые обладают свойством (3), называемый нами классом сферически симметричных уравнений. Следующее утверждение дает описание класса $\mathfrak{K}_{1}\left(\mathbb{R}^{3}\right)$.

Теорема 1. Для того чтобы функиия $S_{j k}[\boldsymbol{v}]$ удовлетворяла уравнению (3), необходимо и достаточно, чтобы она имела вид

$$
S_{j k}[\boldsymbol{v}]=f\left(\boldsymbol{v}^{2}\right) \delta_{j k}+g\left(\boldsymbol{v}^{2}\right) v_{j} v_{k},
$$

где $f, g-\oint у н к и и и ~ н а ~ \mathbb{R}_{+}$. 
Доказательство. Формула (4) при $\mathrm{U}=\mathbf{1}$ превращается в тождество. Тогда при произвольном преобразовании $U$ группы, согласно (4), матрица $S_{j k}[\boldsymbol{v}]$ переходит в $U_{j m} U_{k l} S_{m l}[\boldsymbol{v}]$. Следовательно, $S_{j k}[\boldsymbol{v}]$ преобразуется как тензор (ковариантный) второго ранга. Представим этот тензор в виде разложения по какому-либо базису $w_{j k}^{(a)}[\boldsymbol{v}], a=1, \ldots, 9$, тензорного представления группы $\mathbb{O}_{3}$ (см., например, [8]),

$$
S_{j k}[\boldsymbol{v}]=\sum_{a=1}^{9} h^{(a)}\left(\boldsymbol{v}^{2}\right) w_{j k}^{(a)}[\boldsymbol{v}]
$$

с коэффициентами $h^{(a)}$, которые являются функциями $h^{(a)}: \mathbb{R}_{+} \mapsto \mathbb{R}$ от единственного инварианта $\boldsymbol{v}^{2}$ вектора $\boldsymbol{v}$, а элементы базиса $w_{j k}^{(a)}[\boldsymbol{v}], a=1, \ldots, 9$, являются, вообще говоря, функциями от $\boldsymbol{v}$. Эти базисные элементы, ввиду произвольности функций $h^{(a)}, a=1, \ldots, 9$, и соотношения (4), могут быть выбраны таким образом, чтобы удовлетворялись соотношения $w_{j k}^{(a)}[\mathbf{U} \boldsymbol{v}]=U_{j m} U_{k l} w_{m l}^{(a)}[\boldsymbol{v}]$. Если элемент базиса не зависит от вектора $\boldsymbol{v}$, то он является инвариантным тензором второго ранга. Существует единственный такой тензор - символ Кронекера $\delta_{j k}$. Если элемент базиса зависит от $\boldsymbol{v}$, то он может быть выбран в виде тензорного произведения вектора $\boldsymbol{v}$ и какого-либо другого вектора. Существует единственное тензорное произведение $w_{j k}^{(a)}=v_{j} v_{k}$, которое удовлетворяет соотношению $w_{j k}^{(a)}[\mathbf{U} \boldsymbol{v}]=U_{j m} U_{k l} w_{m l}^{(a)}[\boldsymbol{v}]$.

Следствие. Класс всех квазилинейных систем класса $\mathfrak{K}_{1}\left(\mathbb{R}^{3}\right)$ описывается формулой

$$
\dot{v}_{j}(\boldsymbol{x}, t)=\left(\nabla_{k}\left[f\left(\boldsymbol{v}^{2}\right) \delta_{j k}+g\left(\boldsymbol{v}^{2}\right) v_{j} v_{k}\right]\right)(\boldsymbol{x}, t), \quad j=1,2,3,
$$

где $f$ и $g$-дифференцируемые функиии на $\mathbb{R}_{+}$.

3. Понятие гиперболичности. Каждой системе (1) квазилинейных уравнений первого порядка, для каждого значения поля $\boldsymbol{v}=\boldsymbol{v}(\boldsymbol{x}, t)$ сопоставим линеаризованную (касательную) систему линейных дифференциальных уравнений первого порядка для вариации $\delta \boldsymbol{v}(\boldsymbol{x}, t)$ векторного поля $\boldsymbol{v}(\boldsymbol{x}, t)$,

$$
\delta \dot{v}_{j}(\boldsymbol{x}, t)=A_{j l}^{(k)}[\boldsymbol{v}] \nabla_{k} \delta v_{l},
$$

где набор матриц $\mathrm{A}^{(k)}, A_{j l}^{(k)}=\left(\mathrm{A}^{(k)}\right)_{j l}, k=1,2,3$, составляет тензор третьего ранга

$$
A_{j l}^{(k)}[\boldsymbol{v}]=\frac{\partial S_{j k}[\boldsymbol{v}]}{\partial v_{l}}=\frac{\partial}{\partial v_{l}}\left[f\left(\boldsymbol{v}^{2}\right) \delta_{j k}+g\left(\boldsymbol{v}^{2}\right) v_{j} v_{k}\right]=2\left[f^{\prime}\left(\boldsymbol{v}^{2}\right) \delta_{j k}+g^{\prime}\left(\boldsymbol{v}^{2}\right) v_{j} v_{k}\right] v_{l}+g\left(\boldsymbol{v}^{2}\right)\left(\delta_{j l} v_{k}+\delta_{k l} v_{j}\right) .
$$

Сопоставим уравнению (6) однородную систему линейных алгебраических уравнений

$$
\left(\omega(\boldsymbol{k}) \delta_{j l}+\sum_{m=1}^{3} k_{m} A_{j l}^{(m)}\right) v_{l}^{(0)}=0, \quad j=1,2,3
$$

относительно компонент вектора $\boldsymbol{v}^{(0)}$, которая получается посредством подстановки $\delta v_{j}=$ $v_{j}^{(0)} \exp (i \omega(\boldsymbol{k}) t-i(\boldsymbol{k}, \boldsymbol{x})), j=1,2,3$ с постоянным вектором $\boldsymbol{v}^{(0)}=\left\langle v_{1}^{(0)}, v_{2}^{(0)}, v_{3}^{(0)}\right\rangle$ в уравнение (6). Здесь $\omega$-постоянная «частота» изменения поля, $\boldsymbol{k}$ - постоянный «волновой вектор». Условием существования ненулевого вектора $\boldsymbol{v}^{(0)}$ является равенство нулю детерминанта

$$
\operatorname{det}\left(\omega(\boldsymbol{k}) \delta_{j l}+\sum_{m=1}^{3} k_{m} A_{j l}^{(m)}\right)=0 .
$$

Это уравнение будем называть спектральным (в теоретической физике оно называется дисперсионным уравнением). Результатом решения этого алгебраического уравнения третьей степени являются функции $\omega_{j}(\boldsymbol{k}), j=1,2,3$ (т. н. дисперсионные зависимости).

Определение 1 (см. [5]). Система (1) называется гиперболической (по Петровскому), если функции $\omega_{j}(\boldsymbol{k}), j=1,2,3$, принимают вещественные значения, и значения каждой пары этих функций не совпадают ни в одной точке $\boldsymbol{k} \in \mathbb{R}^{3}$. 
4. Критерий вещественности корней. В этом разделе мы приведем известный критерий вещественности корней кубического уравнения. Рассмотрим уравнение

$$
z^{3}+a_{1} z^{2}+a_{2} z+a_{3}=0 .
$$

относительно $z \in \mathbb{C}$ с вещественными коэффициентами $a_{1}, a_{2}, a_{3}$. В этом случае имеет место следуюшая теорема.

Теорема 2 (см. [9]). Для того чтобы все корни уравнения (8) были вещественны и различны, необходимо и достаточно, чтобы его дискриминант $\Delta$ был положителен, т.е. для коэфбициентов $a_{1}, a_{2}, a_{3}$ выполнялось неравенство

$$
\Delta\left(a_{1}, a_{2}, a_{3}\right)=a_{1}^{2} a_{2}^{2}-4 a_{2}^{3}-4 a_{1}^{3} a_{3}-27 a_{3}^{2}+18 a_{1} a_{2} a_{3}>0 .
$$

Доказательство. Замена $z=y-a_{1} / 3$ приводит уравнение (9) к виду $y^{3}+p y+q=0$ с коэффициентами

$$
p=a_{2}-\frac{a_{1}^{3}}{3}, \quad q=2\left(\frac{a_{1}}{3}\right)^{3}-\frac{a_{1} a_{2}}{3}+a_{3} .
$$

Решениями этого уравнения являются (см., например, [13])

$$
y_{1}=\alpha+\beta, \quad y_{2,3}=-\frac{1}{2}(\alpha+\beta) \pm \frac{\sqrt{3}}{2}(\alpha-\beta),
$$

где

$$
\alpha=\left(-\frac{q}{2}+\sqrt{Q}\right)^{1 / 3}, \quad \beta=\left(-\frac{q}{2}-\sqrt{Q}\right)^{1 / 3}, \quad Q=\left(\frac{p}{3}\right)^{3}+\left(\frac{q}{2}\right)^{2} .
$$

При этом все решения различны и вещественны, если $Q<0$. Так как дискриминант $\Delta$ связан с $Q$ соотношением $\Delta=-108 Q$, то критерием вещественности и различности корней является $\Delta>0$. Подставляя в выражение для $Q$ явный вид коэффициентов $p$ и $q$, получим неравенство (9).

5. Вычисление дискриминанта. В этом разделе мы вычислим дискриминант $\Delta\left(a_{1}, a_{2}, a_{3}\right)$ спектрального уравнения (7), определяемого левой частью неравенства (9). Уравнение (7) представим в виде (8),

$$
\omega^{3}+a_{1} \omega^{2}+a_{2} \omega+a_{3}=0
$$

где $\omega \equiv \omega(\boldsymbol{k})$ и (см., например, [4])

$$
a_{1}=\mathrm{SpA}, \quad a_{2}=\frac{1}{2}\left[\mathrm{Sp}^{2} \mathrm{~A}-\mathrm{SpA}^{2}\right], \quad a_{3}=\frac{1}{6}\left[2 \mathrm{SpA}^{3}-3 \mathrm{SpA} \cdot \mathrm{SpA}^{2}+\mathrm{Sp}^{3} \mathrm{~A}\right]
$$

с матрицей $\mathrm{A}=\mathrm{A}^{(l)} k_{l}$. Воспользовавшись явным видом (6), представим матрицу А в виде суммы $\mathrm{A}=2 \mathrm{~B}+g \mathrm{C}$ матриц с матричными элементами

$$
B_{j m}=b_{j} v_{m}, \quad C_{j m}=\zeta \delta_{j m}+v_{j} k_{m}, \quad b_{j}=f^{\prime} k_{j}+\zeta g^{\prime} v_{j}, \quad \zeta=(\boldsymbol{k}, \boldsymbol{v}) .
$$

Вычислим следы степеней матрицы $\mathrm{C}: c_{1}=\mathrm{SpC}=4 \zeta$,

$$
\begin{gathered}
\left(\mathrm{C}^{2}\right)_{j l}=\zeta\left(\zeta \delta_{j l}+3 v_{j} k_{l}\right), \quad \mathrm{SpC}^{2}=6 \zeta^{2}, \\
\left(\mathrm{C}^{3}\right)_{j l}=\zeta\left(\zeta \delta_{j m}+3 v_{j} k_{m}\right)\left(\zeta \delta_{l m}+v_{m} k_{l}\right)=\zeta^{2}\left(\zeta \delta_{j l}+7 v_{j} k_{l}\right), \quad \mathrm{Sp} \mathrm{C}^{3}=10 \zeta^{3} .
\end{gathered}
$$

Из (12) и (13) следует, что

$$
c_{2} \equiv\left[\mathrm{Sp}^{2} \mathrm{C}-\mathrm{SpC}^{2}\right] / 2=5 \zeta^{2}, \quad c_{3} \equiv\left[2 \mathrm{Sp} C^{3}-3 \mathrm{SpC} \cdot \mathrm{Sp} C^{2}+\mathrm{Sp}^{3} \mathrm{C}\right] / 6=2 \zeta^{3} .
$$

Кроме того, так как

$$
(\boldsymbol{v}, \boldsymbol{b})=\zeta\left(f^{\prime}+\boldsymbol{v}^{2} g^{\prime}\right), \quad(\boldsymbol{k}, \boldsymbol{b})=f^{\prime} \boldsymbol{k}^{2}+g^{\prime} \zeta^{2}
$$

где $\boldsymbol{b}=\left\langle b_{1}, b_{2}, b_{3}\right\rangle$, то

$$
\begin{gathered}
(\mathrm{C} \boldsymbol{b})_{j}=C_{j l} b_{l}=\zeta b_{j}+v_{j}(\boldsymbol{k}, \boldsymbol{b}), \quad(\boldsymbol{v}, \mathrm{C} \boldsymbol{b})=\zeta(\boldsymbol{v}, \boldsymbol{b})+\boldsymbol{v}^{2}(\boldsymbol{k}, \boldsymbol{b}), \\
\left(\mathrm{C}^{2} \boldsymbol{b}\right)_{j}=\left(\mathrm{C}^{2}\right)_{j l} b_{l}=\zeta\left(\zeta b_{j}+3 v_{j}(\boldsymbol{k}, \boldsymbol{b})\right), \quad\left(\boldsymbol{v}, \mathrm{C}^{2} \boldsymbol{b}\right)=\zeta\left(\zeta(\boldsymbol{v}, \boldsymbol{b})+3 \boldsymbol{v}^{2}(\boldsymbol{k}, \boldsymbol{b})\right) .
\end{gathered}
$$


Теперь мы в состоянии вычислить коэффициенты $a_{1}, a_{2}, a_{3}$ спектрального уравнения (10) по формулам (11). Учитывая разложение матрицы А, находим

$$
a_{1}=\mathrm{SpA}=2 \mathrm{SpB}+c_{1} g=2 d_{0}+c_{1} g, \quad d_{0} \equiv(\boldsymbol{b}, \boldsymbol{v}) .
$$

Далее, поскольку

$$
\mathrm{A}^{2}=(2 \mathrm{~B}+g \mathrm{C})^{2}=4 \mathrm{~B}^{2}+2 g(\mathrm{BC}+\mathrm{CB})+g^{2} \mathrm{C}^{2}, \quad \mathrm{~B}^{2}=(\boldsymbol{b}, \boldsymbol{v}) \mathrm{B}, \quad \mathrm{Sp} \mathrm{B}^{2}=(\boldsymbol{b}, \boldsymbol{v})^{2},
$$

Tо

$$
\begin{gathered}
\left(\mathrm{A}^{2}\right)_{j l}=4(\boldsymbol{b}, \boldsymbol{v}) \mathrm{B}_{j l}+2 g\left(b_{j}\left(\mathrm{C}^{\mathrm{T}} \boldsymbol{v}\right)_{l}+(\mathrm{C} \boldsymbol{b})_{j} v_{l}\right)+g^{2}\left(\mathrm{C}^{2}\right)_{j l}, \\
\mathrm{SpA}^{2}=4 d_{0}^{2}+4 g d_{1}+g^{2} \mathrm{SpC}^{2}, \quad d_{1} \equiv(\boldsymbol{v}, \mathrm{C} \boldsymbol{b}) .
\end{gathered}
$$

Следовательно, согласно (11),

$$
a_{2}=\frac{1}{2}\left[\left(2 d_{0}+c_{1} g\right)^{2}-g^{2} \operatorname{Sp} C^{2}\right]-2\left(d_{0}^{2}+g d_{1}\right)=g^{2} c_{2}+2 g\left(d_{0} c_{1}-d_{1}\right) .
$$

Наконец, вычислим коэффициент $a_{3}$. Так как

$$
\mathrm{A}^{3}=8 \mathrm{~B}^{3}+4 g\left(\mathrm{~B}^{2} \mathrm{C}+\mathrm{BCB}+\mathrm{CB}^{2}\right)+2 g^{2}\left(\mathrm{BC}^{2}+\mathrm{CBC}+\mathrm{C}^{2} \mathrm{~B}\right)+g^{3} \mathrm{C}^{3},
$$

TO

$$
\begin{aligned}
&\left(\mathrm{A}^{3}\right)_{j l}=8(\boldsymbol{b}, \boldsymbol{v})^{2} b_{j} v_{l}+4 g\left[(\boldsymbol{b}, \boldsymbol{v}) b_{j}\left(\mathrm{C}^{\mathrm{T}} \mathbf{a}_{l}\right)+\right.\left.(\boldsymbol{v}, \mathrm{C} \boldsymbol{b}) b_{j} v_{l}+(\boldsymbol{b}, \boldsymbol{v})(\mathrm{C} \boldsymbol{b})_{j} v_{l}\right]+ \\
&+2 g^{2}\left[b_{j}\left(\mathrm{C}^{2 \mathrm{~T}} \boldsymbol{v}\right)_{l}+(\mathrm{C} \boldsymbol{b})_{j}\left(\mathrm{C}^{\mathrm{T}} \boldsymbol{v}\right)_{l}+\left(\mathrm{C}^{2} \boldsymbol{b}\right)_{j} \boldsymbol{v}_{l}\right]+g^{3}\left(\mathrm{C}^{3}\right)_{j l}, \\
& \mathrm{Sp} \mathrm{A}^{3}=8 d_{0}^{3}+12 g d_{0} d_{1}+6 g^{2} d_{2}+g^{3} \mathrm{SpC}^{3}, \quad d_{2}=\left(\boldsymbol{v}, \mathrm{C}^{2} \boldsymbol{b}\right) .
\end{aligned}
$$

Тогда, используя формулы (16), (19), (21), получаем выражение для коэффициента $a_{3}$ на основе его определения (11),

$$
\begin{aligned}
a_{3}= & \operatorname{SpA}^{3}+\operatorname{SpA}\left[a_{2}-\mathrm{SpA}^{2}\right]=\frac{1}{3}\left[8 d_{0}^{3}+12 g d_{0} d_{1}+6 g^{2} d_{2}+g^{3} \mathrm{Sp} \mathrm{C}^{3}\right]+ \\
& +\frac{1}{3}\left(2 d_{0}+c_{1} g\right)\left[g^{2}\left(c_{2}-\mathrm{SpC}^{2}\right)+2 g d_{0} c_{1}-4 d_{0}^{2}-6 g d_{1}\right]=g^{3} c_{3}+2 g^{2}\left(d_{2}+d_{0} c_{2}-d_{1} c_{1}\right)
\end{aligned}
$$

где мы воспользовались тождеством $\mathrm{Sp}^{3}+c_{1}\left(c_{2}-\mathrm{Sp}^{2}\right)=3 c_{3}$.

Выразим коэффициенты $a_{1}, a_{2}, a_{3}$ в терминах функций $f$ и $g$. Следуя формуле $(18)$, находим $d_{0}=\zeta\left(f^{\prime}+\boldsymbol{v}^{2} g^{\prime}\right)$. Далее, на основе $(20)$ и $(15),(16)$, получаем

$$
d_{1}=\zeta^{2}\left(f^{\prime}+2 \boldsymbol{v}^{2} g^{\prime}\right)+\boldsymbol{k}^{2} \boldsymbol{v}^{2} f^{\prime}
$$

точно так же, используя формулы $(22)$ и $(17),(15)$, имеем

Тогда

$$
d_{2}=\zeta\left[\zeta^{2}\left(f^{\prime}+4 \boldsymbol{v}^{2} g^{\prime}\right)+3 \boldsymbol{v}^{2} \boldsymbol{k}^{2} f^{\prime}\right] .
$$

$$
d_{2}+d_{0} c_{2}-d_{1} c_{1}=\zeta\left[2 \zeta(\boldsymbol{b}, \boldsymbol{v})-\boldsymbol{v}^{2}(\boldsymbol{k}, \boldsymbol{b})\right]=\zeta\left[\zeta^{2}\left(2 f^{\prime}+\boldsymbol{v}^{2} g^{\prime}\right)-\boldsymbol{k}^{2} \boldsymbol{v}^{2} f^{\prime}\right]
$$

и, следовательно, на основании (23),

$$
a_{3}=2 \zeta g^{2}\left[\zeta^{2}\left(2 f^{\prime}+\boldsymbol{v}^{2} g^{\prime}+g\right)-\boldsymbol{k}^{2} \boldsymbol{v}^{2} f^{\prime}\right] \equiv|\boldsymbol{k}|^{3}|\boldsymbol{v}|^{3}\left(\eta^{3} e_{3}-\eta r_{3}\right),
$$

где выделена явно зависимость от угла между векторами $\boldsymbol{k}$ и $\boldsymbol{v}, \eta=\cos (\widehat{\boldsymbol{k}, \boldsymbol{v}})$ с коэффициентами $e_{3}=2 g^{2}\left(2 f^{\prime}+v^{2} g^{\prime}+g\right), r_{3}=2 g^{2} f^{\prime}$. Далее, согласно формулам (14) и (21), а также найденным выше выражениям для $d_{0}$ и $d_{1}$, находим аналогичные представления для коэффициентов $a_{2}$ и $a_{1}$

$$
\begin{gathered}
a_{2}=g\left[\zeta^{2}\left(5 g+6 f^{\prime}+4 \boldsymbol{v}^{2} g^{\prime}\right)-2 \boldsymbol{k}^{2} \boldsymbol{v}^{2} f^{\prime}\right] \equiv \boldsymbol{k}^{2} \boldsymbol{v}^{2}\left(\eta^{2} e_{2}-r_{2}\right), \\
e_{2}=g\left(5 g+6 f^{\prime}+4 \boldsymbol{v}^{2} g^{\prime}\right), \quad r_{2}=2 g f^{\prime} ; \\
a_{1}=2 \zeta\left(f^{\prime}+\boldsymbol{v}^{2} g^{\prime}+2 g\right)=|\boldsymbol{k} \| \boldsymbol{v}| \eta e_{1}, \quad e_{1}=2\left(2 g+f^{\prime}+\boldsymbol{v}^{2} g^{\prime}\right) .
\end{gathered}
$$


После подстановки полученных выражений (25)-(27) для коэффициентов $a_{1}, a_{2}, a_{3}$ в (9) приходим к выводу, что справедлива

Теорема 3. Дискриминант $\Delta\left(a_{1}, a_{2}, a_{3}\right)$ уравнения (8) равен

$$
\Delta\left(a_{1}, a_{2}, a_{3}\right)=\boldsymbol{k}^{6} \boldsymbol{v}^{6} \Delta\left(\eta e_{1}, \eta^{2} e_{2}-r_{2}, \eta^{3} e_{3}-\eta r_{3}\right),
$$

где функиии $e_{1}, e_{2}, r_{2}, e_{3}, r_{3}$ даются формулами (24), (26), (27). Он представляет собой бикубический полином по переменной $\eta=\cos (\widehat{\boldsymbol{k}, \boldsymbol{v}})$.

6. Вещественность спектра. Принимая во внимание формулу (28), необходимым и достаточным условием гиперболичности уравнения (5), т.е. вещественности решений $\omega_{j}(\boldsymbol{k}), j=1,2,3$, спектрального уравнения (10) и отсутствия их вырождения, является выполнимость неравенства $\Delta\left(\eta e_{1}, \eta^{2} e_{2}-r_{2}, \eta^{3} e_{3}-\eta r_{3}\right)>0$. Проведем анализ возможности выполнения этого неравенства для бикубического по $\eta$ полинома.

Рассмотрим при $u \in \mathbb{R}$ кубический полином $P(u)=\alpha_{0} u^{3}+\alpha_{1} u^{2}+\alpha_{2} u+\alpha_{3}$ и исследуем, какие возникают ограничения на коэффициенты $\alpha_{0}, \alpha_{1}, \alpha_{2}, \alpha_{3}$ этого полинома в том случае, когда для него выполняется неравенство $P(u)>0$ на интервале $[0,1]$. Очевидно, что для этого необходимо, чтобы $P(0)>0$ и $P(1)>0$. Эти же условия являются и достаточными в том случае, если на $(0,1)$ не имеется такой точки $u_{*}$, что $P^{\prime}\left(u_{*}\right)=0, P^{\prime \prime}\left(u_{*}\right)>0$, где $P^{\prime}(u)=3 \alpha_{0} u^{2}+2 \alpha_{1} u+$ $\alpha_{2}, P^{\prime \prime}(u)=6 \alpha_{0}+2 \alpha_{1}$. Если существует такая точка, то необходимо, чтобы $P\left(u_{*}\right)>0$ и это условие, в сочетании с неравенствами $P(0)>0, P(1)>0$, составляет полный набор необходимых и достаточных условий. Изучим представляющиеся при этом возможности.

Будем различать следующие случаи:

I. $\alpha_{0}=0$;

II. $\alpha_{0} \neq 0, \alpha_{1}^{2} \leqslant 3 \alpha_{0} \alpha_{2}$;

III. $\alpha_{0} \neq 0, \alpha_{1}^{2}>3 \alpha_{0} \alpha_{2}$.

В первом случае $P(u)=2 \alpha_{1}, u_{*}=-\alpha_{2} / 2 \alpha_{1}$. Тогда, если $\alpha_{1}=0$, то точка $u_{*}$ заведомо не существует. Если $\alpha_{1} \neq 0$ и существует точка $u_{*} \in(0,1)$, то $-\alpha_{2} / 2 \alpha_{1} \in(0,1)$. При этом ввиду условия $P\left(u_{*}\right)>0$ получаем, что $\alpha_{1}>0$. Следовательно, в этом случае $\alpha_{2}<0$ и $\alpha_{2}+2 \alpha_{1}>0$. Если точка $u_{*}$ не принадлежит $(0,1)$, то либо $\alpha_{1}<0$ и имеет место по крайней мере одно из неравенств $\alpha_{2} \leqslant 0, \alpha_{2}+2 \alpha_{1} \leqslant 0$, либо $\alpha_{1}>0$ и выполняется одно из неравенств $\alpha_{2} \geqslant 0, \alpha_{2}+2 \alpha_{1} \geqslant 0$. Наконец, если точка $u_{*}$ находится на интервале $(0,1)$, то $\alpha_{1}>0$ и одновременно имеют место $\alpha_{2}<0, \alpha_{1}+2 \alpha_{2}>0$.

В случае II точка $u_{*}$ заведомо отсутствует (в случае $\alpha_{1}^{2} \leqslant 3 \alpha_{0} \alpha_{2}, u_{*}=-\alpha_{1} / 3 \alpha_{0}$ и $P^{\prime \prime}\left(u_{*}\right)=0$, т.е. $u_{*}$ не является точкой минимума).

В случае III существование точки $u_{*}$ возможно, и она может принимать одно из двух значений

$$
u_{ \pm}=\frac{1}{3 \alpha_{0}}\left(-\alpha_{1} \pm R\right), \quad R=\sqrt{\alpha_{1}^{2}-3 \alpha_{2} \alpha_{0}} .
$$

Если $u_{*} \in(0,1)$, то условию $P\left(u_{*}\right)>0$ нужно удовлетворить только при $u_{*}=u_{+}$, так как $P^{\prime \prime}\left(u_{ \pm}\right)= \pm 2 R$, и для точки $u_{-}$неравенство $P^{\prime \prime}\left(u_{*}\right)>0$ невозможно. Таким образом, нужно изучить только возможность принадлежности $u_{+} \in(0,1)$, так как если $u_{+} \notin(0,1)$, то условия $P(0)>0$ и $P(1)>0$ являются достаточными для выполнимости неравенства $P(u)>0$ при $u \in[0,1]$. Рассмотрим два случая $\mathrm{III}_{ \pm}$, в зависимости от выбора знака у коэффициента $\alpha_{0}$.

В случае $\mathrm{III}_{+}$при $\alpha_{0}>0$, для того чтобы $u_{+} \in(0,1)$, необходимо и достаточно, чтобы $\alpha_{1}<R<$ $\alpha_{1}+3 \alpha_{0}$. Тогда для выполнения левого неравенства необходимо и достаточно, чтобы выполнялись либо $\alpha_{1} \leqslant 0$, либо $\alpha_{1}>0, \alpha_{2}>0$. Правое же неравенство выполняется в том и только в том случае, если $3 \alpha_{0}+\alpha_{1}>0, \alpha_{2}+2 \alpha_{1}+3 \alpha_{0}>0$.

В случае III_ неравенство $0<u_{+}<1$ удовлетворяется в двух случаях: либо при $R<\alpha_{1}$, либо при $3 \alpha_{0}+\alpha_{1}<R$. Первое неравенство дает $\alpha_{1}>0$ и $\alpha_{2}<0$. Выполнимость второго неравенства возможна только либо при $3 \alpha_{0}+\alpha_{1} \leqslant 0$, либо при $3 \alpha_{0}+\alpha_{1}>0$ и $3 \alpha_{0}+2 \alpha_{1}+\alpha_{2}>0$.

Исследуем возможность реализации неравенства $P\left(u_{+}\right)>0$ при $u_{+} \in(0,1)$ в случае III. Так как имеет место равенство

$$
3 P(u)=\left(3 u+\alpha_{1}\right) P^{\prime}(u)+2 u\left(3 \alpha_{0} \alpha_{2}-\alpha_{1}^{2}\right)+9 \alpha_{3}-\alpha_{1} \alpha_{2},
$$


то на основании условий $P\left(u_{+}\right)>0, P^{\prime}\left(u_{+}\right)=0$ находим

$$
2 u_{+} R^{2}<9 \alpha_{3}-\alpha_{1} \alpha_{2} .
$$

Для того чтобы имело место $P\left(u_{+}\right)>0$ при $u_{+} \in(0,1)$, необходимо и достаточно, чтобы $2 u_{+} R<9 \alpha_{3}-\alpha_{1} \alpha_{2}$, и поэтому $9 \alpha_{3}-\alpha_{1} \alpha_{2}>0$. Отсюда следует, что при выполнении последнего неравенства необходимо и достаточно, чтобы в случае $\mathrm{III}_{+}$имело место неравенство $2 R^{3}<S$, где $S \equiv 3 \alpha_{0}\left(9 \alpha_{3}-\alpha_{1} \alpha_{2}\right)+2 \alpha_{1} R^{2}=27 \alpha_{0} \alpha_{3}-9 \alpha_{0} \alpha_{1} \alpha_{2}+2 \alpha_{1}^{3}$, а в случае III_ имело место обратное неравенство $2 R^{3}>S$. Проанализируем эти возможности.

В случае $\mathrm{III}_{+}$обязательно должно выполняться $S>0$. Тогда запишем неравенство $2 R^{3}<$ $S$ в эквивалентной форме $4 R^{6}<S^{2}$, которая не содержит радикалов. Последнее неравенство преобразуется с учетом $\alpha_{0}>0$ к виду

$$
4 \alpha_{0}^{2} \alpha_{2}^{3}+\alpha_{0}\left(27 \alpha_{3}^{2}-18 \alpha_{1} \alpha_{2} \alpha_{3}-\alpha_{1}^{2} \alpha_{2}^{2}\right)+4 \alpha_{1}^{3} \alpha_{3}>0
$$

В случае III_ неравенство $2 R^{3}>S$ всегда выполняется при $S<0$, а при $S>0$ оно эквивалентно неравенству $4 R^{6}>S^{2}$, не содержащему радикалов. Последнее после деления на $\alpha_{0}<0$ превращается снова в неравенство (29). Результаты проведенного анализа сформулируем в виде отдельного утверждения.

Лемма 1. Для того чтобъ значения кубического полинома $P(u)=\alpha_{0} u^{3}+\alpha_{1} u^{2}+\alpha_{2} u+\alpha_{3}$ бъли строго положстельны на отрезке [0,1], необходимо и достаточно, чтобы:

$$
P(0)=\alpha_{3}>0, \quad P(1)=\alpha_{0}+\alpha_{2}+\alpha_{2}+\alpha_{3}>0,
$$

а такэе выполнялись условия, перечисленные по крайней мере в одном из пn. I-III.

I. $Е с л и \alpha_{0}=0$, mo

$$
\begin{aligned}
& \text { либо } \alpha_{1}=0 ; \\
& \text { либо } \alpha_{1}<0 \text { и }\left(\alpha_{2} \leqslant 0 \text { или } \alpha_{2}+2 \alpha_{1} \leqslant 0\right) ; \\
& \text { либо } \alpha_{1}>0 \text { и }\left(\alpha_{2} \geqslant 0 \text { или } \alpha_{2}+2 \alpha_{1} \geqslant 0\right) ; \\
& \text { либо одновременно } \alpha_{1}>0, \alpha_{2}>0, \alpha_{2}+2 \alpha_{1}>0 .
\end{aligned}
$$

II. $\alpha_{0} \neq 0, \alpha_{1}^{2} \leqslant 3 \alpha_{0} \alpha_{2}$.

III. Если $\alpha_{0} \neq 0, \alpha_{1}^{2}>\alpha_{0} \alpha_{2}$, то в случае $\mathrm{III}_{+}$, когда $\alpha_{0}>0$, должсна реализоваться одна из следующих возможностей:

(i) $\alpha_{1}>0, \alpha_{2} \leqslant 0$ или нарушается по крайней мере одно из неравенств

$$
3 \alpha_{0}+\alpha_{1}>0, \quad \alpha_{2}+2 \alpha_{1}+3 \alpha_{0}>0
$$

(ii) $\alpha_{1}>0$ и $\alpha_{2}>0$, а также одновременно выполняются неравенства

$$
3 \alpha_{0}+\alpha_{1}>0, \quad \alpha_{2}+2 \alpha_{1}+3 \alpha_{0}>0, \quad 9 \alpha_{3}>\alpha_{1} \alpha_{2}, \quad 27 \alpha_{0} \alpha_{3}-9 \alpha_{0} \alpha_{1} \alpha_{2}+2 \alpha_{1}^{3}>0
$$

и неравенство (29).

В случае же III-, когда $\alpha_{0}<0$, должна реализоваться одна из следующих возможноcmeй:

(i) Выполняется по крайней мере одно из неравенств $\alpha_{1} \leqslant 0 ; \alpha_{2} \geqslant 0$;

$$
3 \alpha_{0}+\alpha_{1}>0, \quad \alpha_{2}+2 \alpha_{1}+3 \alpha_{0} \leqslant 0
$$

(ii) Одновременно выполняются условия $\alpha_{1}>0 u \alpha_{2}>0$, а также реализуется либо $3 \alpha_{0}+\alpha_{1} \leqslant 0$, либо

$$
3 \alpha_{0}+\alpha_{1}>0, \quad \alpha_{2}+2 \alpha_{1}+3 \alpha_{0}>0
$$

Кроме того, вместе с неравенством (29) долэни выполняться неравенства

$$
9 \alpha_{3}>\alpha_{1} \alpha_{2}, \quad 27 \alpha_{0} \alpha_{3}-9 \alpha_{0} \alpha_{1} \alpha_{2}+2 \alpha_{1}^{3}<0
$$


Применим теперь результаты анализа неравенства $P(u)>0$ на $[0,1]$ к описанию области выполнимости неравенства

$$
\Delta\left(\eta e_{1}, \eta^{2} e_{2}-r_{2}, \eta^{3} e_{3}-\eta r_{3}\right)>0 .
$$

С этой целью разложим $\Delta\left(\eta e_{1}, \eta^{2} e_{2}-r_{2}, \eta^{3} e_{3}-\eta r_{3}\right)$ по степеням $\eta$. Заметим, что дискриминант (9) обладает тем свойством, что для любого $\lambda \in \mathbb{R}$ имеет место равенство

$$
\Delta\left(\lambda a_{1}, \lambda^{2} a_{2}, \lambda^{3} a_{3}\right)=\lambda^{6} \Delta\left(a_{1}, a_{2}, a_{3}\right) .
$$

Тогда справедливо тождество

$$
\Delta\left(\eta e_{1}, \eta^{2} e_{2}-r_{2}, \eta^{3} e_{3}-\eta r_{3}\right)=\eta^{6} \Delta\left(e_{1}, e_{2}-\eta^{-2} r_{2}, e_{3}-\eta^{-2} r_{3}\right) .
$$

Искомое разложение получается разложением в ряд Тейлора функции $\Delta\left(e_{1}, e_{2}-\eta^{-2} r_{2}, e_{3}-\eta^{-2} r_{3}\right)$ по степеням $\eta^{-2}$, которое содержит четыре слагаемых. Тогда

$$
\Delta\left(\eta e_{1}, \eta^{2} e_{2}-r_{2}, \eta^{3} e_{3}-\eta r_{3}\right)=\eta^{6}\left(\Delta\left(e_{1}, e_{2}, e_{3}\right)+\eta^{-2} \Delta_{1}+\eta^{-4} \Delta_{2}+\eta^{-6} \Delta_{3}\right),
$$

где, используя явный вид $\Delta\left(a_{1}, a_{2} . a_{3}\right)$ и явные выражения для функций $r_{2}$ и $r_{3}$, находим, что

$$
\begin{aligned}
\Delta_{1} & =-r_{2}\left(\frac{\partial \Delta}{\partial a_{2}}\right)_{a_{2}=e_{2}}-r_{3}\left(\frac{\partial \Delta}{\partial a_{3}}\right)_{a_{3}=e_{3}}= \\
& =4 g f^{\prime}\left[6 e_{2}^{2}-9 e_{1} e_{3}-e_{1}^{2} e_{2}+g\left(2 e_{1}^{3}+27 e_{3}-9 e_{1} e_{2}\right)\right], \\
\Delta_{2} & =\frac{1}{2}\left(\frac{\partial^{2} \Delta}{\partial a_{2}^{2}} r_{2}^{2}+2 \frac{\partial^{2} \Delta}{\partial a_{2} \partial a_{3}} r_{2} r_{3}+\frac{\partial^{2} \Delta}{\partial a_{3}^{2}} r_{3}^{2}\right)_{a_{2}=e_{2}, a_{3}=r_{3}}= \\
& =4 g^{2} f^{\prime 2}\left[e_{1}^{2}-12 e_{2}-27 g^{2}+18 g e_{1}\right]= \\
& =4 g^{2} f^{\prime 2}\left[g^{2}+4\left(f^{\prime 2}+\boldsymbol{v}^{2} g g^{\prime}+\boldsymbol{v}^{4} g^{\prime 2}\right)+8 \boldsymbol{v}^{2} f^{\prime} g^{\prime}-20 g f^{\prime}\right], \\
\Delta_{3} & =-\frac{1}{6}\left(\frac{\partial^{3} \Delta}{\partial a_{2}^{3}}\right)_{a_{2}=e_{2}} r_{2}^{3}=32 g^{3} f^{\prime 3} .
\end{aligned}
$$

Подстановкой явных выражений для функций $e_{1}, e_{2}, e_{3}$ получаем следующие формулы для $\Delta_{1}$ и $\Delta_{2}$ :

$$
\begin{aligned}
& \Delta_{1}=8 g^{2} f^{\prime 2}\left[\left(8 f^{\prime}-4 \boldsymbol{v}^{2} g^{\prime}-g\right) g-4\left(f^{\prime}+\boldsymbol{v}^{2} g^{\prime}\right)^{2}\right] \\
& \Delta_{2}=4 g^{2}{f^{\prime}}^{2}\left[\left(g+4 \boldsymbol{v}^{2} g^{\prime}-20 f^{\prime}\right) g+4\left(f^{\prime}+\boldsymbol{v}^{2} g^{\prime}\right)^{2}\right] .
\end{aligned}
$$

При этом

$$
\begin{gathered}
2 \Delta_{1}+\Delta_{2}=12 g^{2} f^{\prime 2}\left[\left(4 f^{\prime}-4 \boldsymbol{v}^{2} g^{\prime}-g\right) g-4\left(f^{\prime}+\boldsymbol{v}^{2} g^{\prime}\right)^{2}\right] \\
\Delta_{1}+\Delta_{2}+\Delta_{3}=4 g^{2}{f^{\prime}}^{2}\left[\left(4 f^{\prime}-4 \boldsymbol{v}^{2} g^{\prime}-g\right) g-4\left(f^{\prime}+\boldsymbol{v}^{2} g^{\prime}\right)^{2}\right] .
\end{gathered}
$$

Положим теперь $u=\eta^{2}, \alpha_{0}=\Delta\left(e_{1}, e_{2}, e_{3}\right), \alpha_{1}=\Delta_{1}, \alpha_{2}=\Delta_{2}, \alpha_{3}=\Delta_{3}$ и воспользуемся формулами

$$
\alpha_{0}+\alpha_{1}+\alpha_{2}+\alpha_{3}=\Delta\left(e_{1}, e_{2}-r_{2}, e_{3}-r_{3}\right)=\Delta\left(e_{1}, e_{2}-2 g f^{\prime}, e_{3}-2 g^{2} f^{\prime}\right) .
$$

В результате на основании леммы убеждаемся в справедливости следующего утверждения, доказательство которого составляет цель настоящей работы.

Теорема 4. Для того чтобы уравнение (1) с тензор-функиией (4) представляло собой систему гиперболических по Петровскому квазилинейных уравнений первого порядка, необходимо и достаточно, чтобы выполнялись неравенства

$$
g f^{\prime}>0, \quad \Delta\left(e_{1}, e_{2}-2 g f^{\prime}, e_{3}-2 g^{2} f^{\prime}\right)>0,
$$

а также условия, перечисленные по крайней мере в одном из следующих пn. I-III: 
I. $\operatorname{Eсли~} \Delta\left(e_{1}, e_{2}, e_{3}\right)=0$, mo

либо $\left(8 f^{\prime}-4 \boldsymbol{v}^{2} g^{\prime}-g\right) g-4\left(f^{\prime}+\boldsymbol{v}^{2} g^{\prime}\right)^{2}=0 ;$

либо $\left(8 f^{\prime}-4 \boldsymbol{v}^{2} g^{\prime}-g\right) g-4\left(f^{\prime}+\boldsymbol{v}^{2} g^{\prime}\right)^{2}<0$

$$
u\left(\left(g+4 \boldsymbol{v}^{2} g^{\prime}-20 f^{\prime}\right) g+4\left(f^{\prime}+\boldsymbol{v}^{2} g^{\prime}\right)^{2} \leqslant 0 \text { или }\left(4 f^{\prime}-4 \boldsymbol{v}^{2} g^{\prime}-g\right) g-4\left(f^{\prime}+\boldsymbol{v}^{2} g^{\prime}\right)^{2} \leqslant 0\right) ;
$$

либо $\left(8 f^{\prime}-4 \boldsymbol{v}^{2} g^{\prime}-g\right) g-4\left(f^{\prime}+\boldsymbol{v}^{2} g^{\prime}\right)^{2}>0$

$$
u\left(\left(g+4 \boldsymbol{v}^{2} g^{\prime}-20 f^{\prime}\right) g+4\left(f^{\prime}+\boldsymbol{v}^{2} g^{\prime}\right)^{2} \geqslant 0 \text { или }\left(4 f^{\prime}-4 \boldsymbol{v}^{2} g^{\prime}-g\right) g-4\left(f^{\prime}+\boldsymbol{v}^{2} g^{\prime}\right)^{2} \geqslant 0\right) ;
$$

либо одновременно

$$
\begin{gathered}
\left(8 f^{\prime}-4 \boldsymbol{v}^{2} g^{\prime}-g\right) g-4\left(f^{\prime}+\boldsymbol{v}^{2} g^{\prime}\right)^{2}>0, \\
\left(g+4 \boldsymbol{v}^{2} g^{\prime}-20 f^{\prime}\right) g+4\left(f^{\prime}+\boldsymbol{v}^{2} g^{\prime}\right)^{2}>0, \\
\left(4 f^{\prime}-4 \boldsymbol{v}^{2} g^{\prime}-g\right) g-4\left(f^{\prime}+\boldsymbol{v}^{2} g^{\prime}\right)^{2}>0 .
\end{gathered}
$$

II. $\operatorname{Ecлu~} \Delta\left(e_{1}, e_{2}, e_{3}\right) \neq 0$, mo

$$
\begin{aligned}
16 g^{2} f^{\prime 2}\left[\left(8 f^{\prime}-4 \boldsymbol{v}^{2} g^{\prime}-g\right) g-4\left(f^{\prime}\right.\right. & \left.\left.+\boldsymbol{v}^{2} g^{\prime}\right)^{2}\right]^{2} \leqslant \\
& \leqslant 3 \Delta\left(e_{1}, e_{2}, e_{3}\right)\left[\left(g+4 \boldsymbol{v}^{2} g^{\prime}-20 f^{\prime}\right) g+4\left(f^{\prime}+\boldsymbol{v}^{2} g^{\prime}\right)^{2}\right]
\end{aligned}
$$

III. $\operatorname{Eсли~} \Delta\left(e_{1}, e_{2}, e_{3}\right) \neq 0$,

$$
16 g^{2} f^{\prime 2}\left[\left(8 f^{\prime}-4 \boldsymbol{v}^{2} g^{\prime}-g\right) g-4\left(f^{\prime}+\boldsymbol{v}^{2} g^{\prime}\right)^{2}\right]^{2}>3 \Delta\left(e_{1}, e_{2}, e_{3}\right)\left[\left(g+4 \boldsymbol{v}^{2} g^{\prime}-20 f^{\prime}\right) g+4\left(f^{\prime}+\boldsymbol{v}^{2} g^{\prime}\right)^{2}\right]
$$

то в случае $\mathrm{III}_{+}$, когда $\Delta\left(e_{1}, e_{2}, e_{3}\right)>0$, долюна реализоваться одна из следующих возможсностей:

(i) $\left(8 f^{\prime}-4 \boldsymbol{v}^{2} g^{\prime}-g\right) g-4\left(f^{\prime}+\boldsymbol{v}^{2} g^{\prime}\right)^{2}>0,\left(g+4 \boldsymbol{v}^{2} g^{\prime}-20 f^{\prime}\right) g+4\left(f^{\prime}+\boldsymbol{v}^{2} g^{\prime}\right)^{2} \leqslant 0$ или нарушается по крайней мере одно из неравенств

$$
\begin{aligned}
& 3 \Delta\left(e_{1}, e_{2}, e_{3}\right)+8\left(g f^{\prime}\right)^{2}\left[\left(8 f^{\prime}-4 \boldsymbol{v}^{2} g^{\prime}-g\right) g-4\left(f^{\prime}+\boldsymbol{v}^{2} g^{\prime}\right)^{2}\right]>0 \\
& 3 \Delta\left(e_{1}, e_{2}, e_{3}\right)+12\left(g f^{\prime}\right)^{2}\left[\left(4 f^{\prime}-4 \boldsymbol{v}^{2} g^{\prime}-g\right) g-4\left(f^{\prime}+\boldsymbol{v}^{2} g^{\prime}\right)^{2}\right]>0 .
\end{aligned}
$$

(ii) $\left(8 f^{\prime}-4 \boldsymbol{v}^{2} g^{\prime}-g\right) g-4\left(f^{\prime}+\boldsymbol{v}^{2} g^{\prime}\right)^{2}>0 u\left(g+4 \boldsymbol{v}^{2} g^{\prime}-20 f^{\prime}\right) g+4\left(f^{\prime}+\boldsymbol{v}^{2} g^{\prime}\right)^{2}>0$, а такэе одновременно выполняются неравенства

$$
\begin{gathered}
3 \Delta\left(e_{1}, e_{2}, e_{3}\right)+8\left(g f^{\prime}\right)^{2}\left[\left(8 f^{\prime}-4 \boldsymbol{v}^{2} g^{\prime}-g\right) g-4\left(f^{\prime}+\boldsymbol{v}^{2} g^{\prime}\right)^{2}\right]>0, \\
3 \Delta\left(e_{1}, e_{2}, e_{3}\right)+12\left(g f^{\prime}\right)^{2}\left[\left(4 f^{\prime}-4 \boldsymbol{v}^{2} g^{\prime}-g\right) g-4\left(f^{\prime}+\boldsymbol{v}^{2} g^{\prime}\right)^{2}\right]>0, \\
9>g f^{\prime}\left[\left(8 f^{\prime}-4 \boldsymbol{v}^{2} g^{\prime}-g\right) g-4\left(f^{\prime}+\boldsymbol{v}^{2} g^{\prime}\right)^{2}\right]\left[\left(g+4 \boldsymbol{v}^{2} g^{\prime}-20 f^{\prime}\right) g+4\left(f^{\prime}+\boldsymbol{v}^{2} g^{\prime}\right)^{2}\right], \\
9 \Delta\left(e_{1}, e_{2}, e_{3}\right)\left(3-\left(g f^{\prime}\right)\left[\left(8 f^{\prime}-4 \boldsymbol{v}^{2} g^{\prime}-g\right) g-4\left(f^{\prime}+\boldsymbol{v}^{2} g^{\prime}\right)^{2}\right] \times\right. \\
\left.\times\left[\left(g+4 \boldsymbol{v}^{2} g^{\prime}-20 f^{\prime}\right) g+4\left(f^{\prime}+\boldsymbol{v}^{2} g^{\prime}\right)^{2}\right]\right)+ \\
+32\left(g f^{\prime}\right)^{3}\left[\left(8 f^{\prime}-4 \boldsymbol{v}^{2} g^{\prime}-g\right) g-4\left(f^{\prime}+\boldsymbol{v}^{2} g^{\prime}\right)^{2}\right]^{3}>0,
\end{gathered}
$$


и вытекающее из (29) неравенство

$$
\begin{aligned}
& \Delta^{2}\left(e_{1}, e_{2}, e_{3}\right)\left[\left(g+4 \boldsymbol{v}^{2} g^{\prime}-20 f^{\prime}\right) g+4\left(f^{\prime}+\boldsymbol{v}^{2} g^{\prime}\right)^{2}\right]^{3}+ \\
& +4 \Delta\left(e_{1}, e_{2}, e_{3}\right)\left(27-18\left(g f^{\prime}\right)\left[\left(8 f^{\prime}-4 \boldsymbol{v}^{2} g^{\prime}-g\right) g-4\left(f^{\prime}+\boldsymbol{v}^{2} g^{\prime}\right)^{2}\right]\left[\left(g+4 \boldsymbol{v}^{2} g^{\prime}-20 f^{\prime}\right) g+\right.\right. \\
& \left.+4\left(f^{\prime}+\boldsymbol{v}^{2} g^{\prime}\right)^{2}\right]-\left(g f^{\prime}\right)^{2}\left[\left(8 f^{\prime}-4 \boldsymbol{v}^{2} g^{\prime}-g\right) g-4\left(f^{\prime}+\boldsymbol{v}^{2} g^{\prime}\right)^{2}\right]^{2}\left[\left(g+4 \boldsymbol{v}^{2} g^{\prime}-20 f^{\prime}\right) g+\right. \\
& \left.\left.\quad+4\left(f^{\prime}+\boldsymbol{v}^{2} g^{\prime}\right)^{2}\right]^{2}\right)+4^{4}\left(g f^{\prime}\right)^{3}\left[\left(8 f^{\prime}-4 \boldsymbol{v}^{2} g^{\prime}-g\right) g-4\left(f^{\prime}+\boldsymbol{v}^{2} g^{\prime}\right)^{2}\right]^{3}>0
\end{aligned}
$$

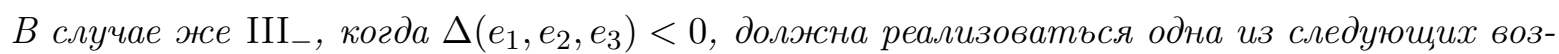
можностей:

(1) Выполняется по крайней мере одно из неравенств

$$
\begin{gathered}
\left(8 f^{\prime}-4 \boldsymbol{v}^{2} g^{\prime}-g\right) g-4\left(f^{\prime}+\boldsymbol{v}^{2} g^{\prime}\right)^{2} \leqslant 0 ; \\
\left(g+4 \boldsymbol{v}^{2} g^{\prime}-20 f^{\prime}\right) g+4\left(f^{\prime}+\boldsymbol{v}^{2} g^{\prime}\right)^{2} \geqslant 0 ; \\
3 \Delta\left(e_{1}, e_{2}, e_{3}\right)+8\left(g f^{\prime}\right)^{2}\left[\left(8 f^{\prime}-4 \boldsymbol{v}^{2} g^{\prime}-g\right) g-4\left(f^{\prime}+\boldsymbol{v}^{2} g^{\prime}\right)^{2}\right]>0 ; \\
3 \Delta\left(e_{1}, e_{2}, e_{3}\right)+12\left(g f^{\prime}\right)^{2}\left[\left(4 f^{\prime}-4 \boldsymbol{v}^{2} g^{\prime}-g\right) g-4\left(f^{\prime}+\boldsymbol{v}^{2} g^{\prime}\right)^{2}\right] \leqslant 0 .
\end{gathered}
$$

(2) $\left(8 f^{\prime}-4 \boldsymbol{v}^{2} g^{\prime}-g\right) g-4\left(f^{\prime}+\boldsymbol{v}^{2} g^{\prime}\right)^{2}>0 u\left(g+4 \boldsymbol{v}^{2} g^{\prime}-20 f^{\prime}\right) g+4\left(f^{\prime}+\boldsymbol{v}^{2} g^{\prime}\right)^{2}>0$, а такэне реализуется либо

$$
3 \Delta\left(e_{1}, e_{2}, e_{3}\right)+8\left(g f^{\prime}\right)^{2}\left[\left(8 f^{\prime}-4 \boldsymbol{v}^{2} g^{\prime}-g\right) g-4\left(f^{\prime}+\boldsymbol{v}^{2} g^{\prime}\right)^{2}\right] \leqslant 0
$$

либо

$$
\begin{aligned}
& 3 \Delta\left(e_{1}, e_{2}, e_{3}\right)+8\left(g f^{\prime}\right)^{2}\left[\left(8 f^{\prime}-4 \boldsymbol{v}^{2} g^{\prime}-g\right) g-4\left(f^{\prime}+\boldsymbol{v}^{2} g^{\prime}\right)^{2}\right] \leqslant 0 \\
& 3 \Delta\left(e_{1}, e_{2}, e_{3}\right)+12\left(g f^{\prime}\right)^{2}\left[\left(4 f^{\prime}-4 \boldsymbol{v}^{2} g^{\prime}-g\right) g-4\left(f^{\prime}+\boldsymbol{v}^{2} g^{\prime}\right)^{2}\right]>0 .
\end{aligned}
$$

Кроме того, должсны выполняться неравенства (30) и

$$
\begin{aligned}
& 9 \Delta\left(e_{1}, e_{2}, e_{3}\right)\left(3-\left(g f^{\prime}\right)\left[\left(8 f^{\prime}-4 \boldsymbol{v}^{2} g^{\prime}-g\right) g-4\left(f^{\prime}+\boldsymbol{v}^{2} g^{\prime}\right)^{2}\right] \times\right. \\
& \left.\quad \times\left[\left(g+4 \boldsymbol{v}^{2} g^{\prime}-20 f^{\prime}\right) g+4\left(f^{\prime}+\boldsymbol{v}^{2} g^{\prime}\right)^{2}\right]\right)+32\left(g f^{\prime}\right)^{3}\left[\left(8 f^{\prime}-4 \boldsymbol{v}^{2} g^{\prime}-g\right) g-4\left(f^{\prime}+\boldsymbol{v}^{2} g^{\prime}\right)^{2}\right]^{3}<0
\end{aligned}
$$

вместе с неравенством (31).

7. Гиперболичность по Фридрихсу. Ввиду технической сложности установления необходимых и достаточных условий гиперболичности систем квазилинейных уравнений первого порядка даже в том относительно простом случае, который был исследован выше, часто объектом исследования систем квазилинейных уравнений первого порядка является установление наличия у них более слабого свойства, чем гиперболичность, а именно, так называемой $t$-гиперболичности (гиперболичности по Фридрихсу) (см. $[5,13])$. Согласно определению понятия $t$-гиперболичности квазилинейных систем первого порядка, для каждой из таких систем существует положительно определенная симметричная матрица $\mathrm{U}^{(0)}$, для которой матрицы $\mathrm{U}^{(0)} \mathrm{U}^{(l)}, l=1,2,3$, являются симметричными. Такое определение понятия $t$-гиперболичности связано со следующим утверждением (ниже мы приводим разъяснения, касающиеся сформулированного утверждения, так как в известной книге [5] в его формулировке, по нашему мнению, допущена существенная неточность).

Теорема 5. Если для системы квазилинейных уравнений первого порядка

$$
\frac{\partial u_{j}}{\partial t}=\sum_{l=1}^{m} \sum_{k=1}^{n} A_{j k}^{(l)} \frac{\partial u_{j}}{\partial x_{l}}, \quad j=1, \ldots, n
$$


относительно набора функций $u_{j}\left(x_{1}, \ldots, x_{m}, t\right), j=1, \ldots, n$, у которой каждая матрица $\mathrm{A}^{(l)}$ из набора матрии $\left(\mathrm{A}^{(l)}\right)_{j k}=A_{j k}^{(l)}, l=1, \ldots, m$, которые зависят от значений этих функиий в текущей точке $\left\langle x_{1}, \ldots, x_{m} ; t\right\rangle$, существует такая положительно определенная симметричная матрица $\mathrm{A}^{(0)},\left(\mathrm{A}^{(0)}\right)_{j k}=A_{j k}^{(0)}$, что матрицы $\left(\mathrm{A}^{(0)} \mathrm{A}^{(l)}\right)_{j k}, l=1, \ldots, m$, являются симметричными, то спектральное уравнение

$$
\operatorname{det}\left(\lambda-\sum_{l=1}^{m} \xi_{l} A_{j k}^{(l)}\right)=0
$$

матриц,

$$
\sum_{l=1}^{m} \xi_{l} A_{j k}^{(l)}
$$

имеет только вещественные решения при любых вещественных наборах $\xi_{l}, l=1, \ldots, m$.

Доказательство. Матрица $\mathrm{A}^{(0)} \mathrm{A}$ симметрична при любом наборе чисел $\xi_{j}, j=1, \ldots, m$,

$$
\mathrm{A}=\sum_{l=1}^{m} \xi_{l} \mathrm{~A}^{(l)}, \quad\left(\mathrm{A}^{(l)}\right)_{j k}=A_{j k}^{(l)} .
$$

Рассмотрим матричный пучок $\lambda \mathrm{A}^{(0)}-\mathrm{A}^{(0)} \mathrm{A}$ с положительно определенной матрицей $\mathrm{A}^{(0)}$. Тогда при любом выборе набора чисел $\xi_{j}, j=1, \ldots, m$, все решения спектрального уравнения $\operatorname{det}\left(\lambda \mathrm{A}^{(0)}-\right.$ $\left.\mathrm{A}^{(0)} \mathrm{A}\right)=0$ для матричного пучка вещественны (см., например, [4]). Поскольку

$$
\operatorname{det} A^{(0)}{ }^{-1} \cdot \operatorname{det}\left(\lambda A^{(0)}-A^{(0)} A\right)=\operatorname{det}(\lambda-A)
$$

т.е. спектральное уравнение (32) пучка совпадает с уравнением $\operatorname{det}(\lambda-\mathrm{A})=0$, заключаем, что все решения этого уравнения также вещественны.

Выясним, какие необходимые и достаточные ограничения на выбор функций $f$ и $g$ необходимо наложить для того, чтобы соответствующее паре этих функций уравнение (5) было $t$-гиперболическим. Для решения поставленной задачи нужно найти такую симметричную положительно определенную матрицу $\mathrm{A}^{(0)}$, для которой матрица

$$
A_{j k}=\sum_{l=1}^{3} k_{l} A_{j m}^{(0)} A_{m k}^{(l)}
$$

симметрична.

Положим, что матрица $\left(\mathrm{A}^{(0)}\right)_{j m}=A_{j m}^{(0)}$ имеет вид $A_{j m}^{(0)}=f^{(0)}\left(\boldsymbol{v}^{2}\right) \delta_{j m}+g^{(0)}\left(\boldsymbol{v}^{2}\right) v_{j} v_{m}$ с некоторыми дифференцируемыми функциями $f^{(0)}$ и $g^{(0)}$ от $\boldsymbol{v}^{2}$. Вычислим соответствующие произведения матриц:

$$
\begin{aligned}
& A_{j m}^{(0)} A_{m k}^{(l)}=\left(f^{(0)} \delta_{j m}+g^{(0)} v_{j} v_{m}\right)\left(2\left[f^{\prime} \delta_{m l}+g^{\prime} v_{m} v_{l}\right] v_{k}+g\left(\delta_{m k} v_{l}+\delta_{k l} v_{m}\right)\right)= \\
& =2 f^{(0)} f^{\prime} \delta_{j l} v_{k}+f^{(0)} g\left(\delta_{j k} v_{l}+\delta_{k l} v_{j}\right)+\boldsymbol{v}^{2} g^{(0)} g v_{j} \delta_{k l}+\left[2\left(f^{(0)} g^{\prime}+g^{(0)} f^{\prime}\right)+g^{(0)}\left(g+2 g^{\prime} \boldsymbol{v}^{2}\right)\right] v_{j} v_{k} v_{l} .
\end{aligned}
$$

Из полученного выражения следует, что матрица $A_{j m}^{(0)} A_{m k}^{(l)}$ симметрична по индексам $j, k$ в том и только в том случае, когда относительно этих индексов симметричен тензор

$$
2 f^{(0)} f^{\prime} \delta_{j l} v_{k}+\left(f^{(0)}+\boldsymbol{v}^{2} g^{(0)}\right) g v_{j} \delta_{l k}
$$

т.е. коэффициенты при линейно независимых тензорах $\delta_{j l} v_{k}$ и $\delta_{l k} v_{j}$ при каждом $l=1,2,3$ совпадают. Отсюда имеем равенство

$$
2 f^{(0)} f^{\prime}=g\left(f^{(0)}+g^{(0)} \boldsymbol{v}^{2}\right) .
$$

Найдем теперь условия, при которых симметричная $3 \times 3$-матрица

$$
A_{j m}^{(0)}=f^{(0)}\left(\boldsymbol{v}^{2}\right) \delta_{j m}+g^{(0)}\left(\boldsymbol{v}^{2}\right) v_{j} v_{m}
$$


является положительно определенной. Для этого вычислим полином $\operatorname{det}\left(\lambda-\mathrm{A}^{(0)}\right)$. Коэффициентами этого полинома являются $\operatorname{Sp}^{(0)}>0,\left(\operatorname{Sp}^{2} A^{(0)}-\operatorname{SpA}^{(0)^{2}}\right) / 2 \operatorname{udet} \mathrm{A}^{(0)}>0$,

$$
\mathrm{SpA}^{(0)}=3 f^{(0)}+\boldsymbol{v}^{2} g^{(0)}>0, \quad \operatorname{det} \mathrm{A}^{(0)}=f^{(0)^{2}}\left(f^{(0)}+\boldsymbol{v}^{2} g^{(0)}\right)>0 .
$$

Так как

$$
\mathrm{Sp} \mathrm{A}^{(0)^{2}}=2 f^{(0)^{2}}+\left(f^{(0)}+\boldsymbol{v}^{2} g^{(0)}\right)^{2}
$$

то на основании (33) второй коэффициент определяется выражением

$$
\frac{1}{2}\left(\mathrm{Sp}^{2} \mathrm{~A}^{(0)}-\mathrm{Sp} \mathrm{A}^{(0)^{2}}\right)=f^{(0)}\left(3 f^{(0)}+2 \boldsymbol{v}^{2} g^{(0)}\right)
$$

и уравнение для собственных чисел матрицы $\mathrm{A}^{(0)}$ принимает вид

$$
\lambda^{3}-\left(3 f^{(0)}+\boldsymbol{v}^{2} g^{(0)}\right) \lambda^{2}+f^{(0)}\left(3 f^{(0)}+2 \boldsymbol{v}^{2} g^{(0)}\right) \lambda-f^{(0)^{2}}\left(f^{(0)}+\boldsymbol{v}^{2} g^{(0)}\right)=0 .
$$

Записав его в виде

$$
\left(\lambda-f^{(0)}\right)^{3}-\boldsymbol{v}^{2} g^{(0)}\left(\lambda-f^{(0)}\right)^{2}=0,
$$

находим собственные числа $\lambda=f^{(0)}, f^{(0)}+\boldsymbol{v}^{2} g^{(0)}$ матрицы $\mathrm{A}^{(0)}$. Таким образом, матрица $\mathrm{A}^{(0)}$ положительно определена при выполнении неравенств $f^{(0)}>0, f^{(0)}+\boldsymbol{v}^{2} g^{(0)}>0$.

Запишем теперь условие (6) в виде

$$
g=2 f^{\prime}\left(1+\frac{\boldsymbol{v}^{2} g^{(0)}}{f^{(0)}}\right)^{-1} .
$$

Так как функции $g^{(0)}$ и $f^{(0)}$ могут быть выбраны произвольно с соблюдением указанных для них ограничений, то, вводя произвольную строго положительную функцию $h\left(\boldsymbol{v}^{2}\right)=2(1+$ $\left.\boldsymbol{v}^{2} g^{(0)} / f^{(0)}\right)^{-1}$, получаем необходимое и достаточное условие $t$-гиперболичности уравнения векторного (5). Таким образом, мы убеждаемся в справедливости следующего утверждения.

Теорема 6. Для того чтобы квазилинейнъе уравнения класса $\mathfrak{K}_{1}\left(\mathbb{R}^{3}\right)$, вся совокупность которых описывается уравнением (5) с произвольными функииями $f\left(\boldsymbol{v}^{2}\right)$ и $g\left(\boldsymbol{v}^{2}\right)$, обладали свойством $t$-гиперболичности, необходимо и достаточно, чтобы $g=h f^{\prime}$, где $h$-произвольная дифференцируемал, строго положительная функиия на $\mathbb{R}_{+}$.

8. Заключение. В работе дано полное описание класса всех гиперболических систем квазилинейных уравнений первого порядка для векторного поля $\boldsymbol{v}(\boldsymbol{x}, t)$, которые допустимо использовать для описания временно́й эволюции этого поля в условиях пренебрежения физическими механизмами диссипации, в частности, энергии. Решение задачи, представленное в работе, показывает, что установление гиперболичности конкретной системы квазилинейных уравнений первого порядка, несмотря на принципиальную разрешимость этой задачи, представляет собой довольно трудоемкий процесс. Более того, несмотря на рутинность предложенного в работе решения и сложность полученного результата, процесс решения удалось довести до конца, не прибегая к каким-либо приближениям. Это связано с тем, что решенная задача является все же довольно простой из всей совокупности такого рода задач, которые могут возникнуть в процессе конструирования физически адекватных математических моделей, когда исследуемые системы дифференциальных уравнений не конкретизированы, а, наоборот, зависят от большого набора параметров, и смысл проводимого анализа как раз и состоит в том, чтобы определить допустимые области изменения этих параметров. В более сложных случаях неизбежно придется прибегать к применению в процессе решения каких-либо приближений. В этом смысле использование более слабого понятия $t$-гиперболичности, которое используется в большинстве исследований задач математической физики, связанных с квазилинейными уравнениями первого порядка (см., например, $[6,7,12])$, может оказаться более предпочтительным. Если дополнительно проводить исследование на возможность пересечения различных ветвей $\omega_{j}(\boldsymbol{k})$ спектральных зависимостей, то, совместно с установлением областей изменения параметров системы, в которых она обладает свойством $t$-гиперболичности, это дает достаточное условие для гиперболичности системы. 


\section{СПИСОК ЛИТЕРАТУРЫ}

1. Вирченко Ю. П., Плесканев А. F. Гиперболические сферически симметричные уравнения первого порядка дивергентного типа для векторного поля// Науч. вед. БелГУ. Сер. мат. физ. - 2019. - 51, № 2. - C. 280-294.

2. Вирченко Ю. П., Субботин А. В. Математические задачи конструирования эволюционных уравнений динамики конденсированных сред// Мат. Междунар. науч. конф. «Дифференциальные уравнения и смежные проблемы» (Стерлитамак, 25-29 июня 2018 г.). - Уфа: Риц БашГУ, 2018. - С. 262-264.

3. Вирченко Ю. П., Субботин А. В. Уравнения динамики конденсированных сред с локальным законом сохранения// Мат. V Междунар. науч. конф. «Нелокальные краевые задачи и родственные проблемы математической биологии, информатики и физики» (Нальчик, 4-7 декабря 2018 г.). — Нальчик: ИПМА КБНЦ РАН, 2018.

4. Гантмахер Ф. Р. Теория матриц. - М.: Физматлит, 2004.

5. Годунов С. К. Уравнения математической физики. - М.: Наука, 1979.

6. Гордиенко В. М. Системы Фридрихса для трехмерного волнового уравнения// Сиб. мат. ж. - 2010. - 51, № 6. - C. 1282-1297.

7. Куликовский А. Г., Погорелов Н. В., Семенов А. Ю. Математические вопросы численного решения гиперболических систем уравнений. - М.: Физматлит, 2001.

8. Любарский Г. Я. Теория групп и ее применения в физике. - М.: Госфизматлит, 1958.

9. Мишина А. П., Проскуряков И. В. Высшая алгебра. - М.: Физматлит, 1962.

10. Рождественский Б. Л., Яненко Н. Н. Системы квазилинейных уравнений и их приложения к газовой динамике. - М.: Наука, 1978.

11. Субботин A. В., Вирченко Ю. П. Описание класса эволюционных уравнений дивергентного типа для векторного поля// Мат. IV Всеросс. науч.-практ. конф. «Современные проблемы физикоматематических наук» (Орел, 22-25 ноября 2018 г.). - Орел: ОГУ им. ни И. С. Тургенева, 2018. - C. $83-86$.

12. Чиркунов Ю. А. Системы фридрихса для систем волновых уравнений и волны сдвига в трехмерной упругой среде// Прикл. мех. техн. физ. - 2010. - 51, № 6. - С. 121-132.

13. Majda A. The existence of multi-dimensional shock fronts// Mem. Am. Math. Soc. — 1983. — 43, № 281 . - P. 1-94.

\section{Вирченко Юрий Петрович}

Белгородский государственный национальный исследовательский университет

E-mail: virch@bsu.edu.ru

Субботин Андрей Валерьевич

Белгородский государственный технологический университет им. В. Г. Шухова

E-mail: subbotin@gmail.com 